\title{
Optimality and duality results for $E$-differentiable multiobjective fractional programming problems under $E$-convexity
}

\author{
Tadeusz Antczak' and Najeeb Abdulaleem²*
}

\begin{tabular}{l}
\hline${ }^{*}$ Correspondence: \\
nabbas985@gmail.com \\
2Department of Mathematics, \\
Hadhramout University, Al-Mahrah, \\
Yemen \\
Full list of author information is \\
available at the end of the article
\end{tabular}

available at the end of the article

\begin{abstract}
A new class of (not necessarily differentiable) multiobjective fractional programming problems with $E$-differentiable functions is considered. The so-called parametric E-Karush-Kuhn-Tucker necessary optimality conditions and, under E-convexity hypotheses, sufficient E-optimality conditions are established for such nonsmooth vector optimization problems. Further, various duality models are formulated for the considered $E$-differentiable multiobjective fractional programming problems and several $E$-duality results are derived also under appropriate $E$-convexity hypotheses.
\end{abstract}

MSC: $90 C 32 ; 90 C 29 ; 90 C 46 ; 49 J 52$

Keywords: E-differentiable multiobjective fractional programming problem; Vector-valued E-convex function; E-optimality conditions; E-duality

\section{Introduction}

In various real-world applications of nonlinear programming more than one ratio of two functions is to be minimized or maximized. Such extremum problems are commonly called multiobjective fractional programming problems. Fractional programming has an important significance in optimization problems. This is a consequence of even the fact that such extremum problems arise from many applied areas including economics, engineering, game theory, numerous decision problems in management science. Therefore, optimality conditions and duality results for various classes of multiobjective fractional programming problems have been of much interest in the recent past (see, for example, [2, 5, 10, 11, 14-21, 23-27, 32-36, 38, 39, 41, 42], and others). Mukherjee and Rao [30] obtained optimality conditions and duality results concerned with differentiable multiobjective fractional nonlinear programming problems under (generalized) $(\rho, b)$-invexity assumptions. In particular, Bector et al. [3] derived Fritz John and Karush-Kuhn-Tucker necessary and sufficient optimality conditions for a class of nondifferentiable convex multiobjective fractional programming problems and established some duality theorems for such problems. Following the approaches of Bector et al. [3], Liu [24, 25] used a parametric approach to obtaining necessary and sufficient conditions and established duality theorems for a class of nonsmooth multiobjective fractional programming problems involving either nonsmooth pseudoinvex functions or nonsmooth $(F, \rho)$-convex functions. Both parametric and nonparametric necessary and sufficient optimality conditions for a class

(c) The Author(s) 2019. This article is distributed under the terms of the Creative Commons Attribution 4.0 International License (http://creativecommons.org/licenses/by/4.0/), which permits unrestricted use, distribution, and reproduction in any medium, provided you give appropriate credit to the original author(s) and the source, provide a link to the Creative Commons license, and indicate if changes were made. 
of nonsmooth generalized fractional programming problems containing $\rho$-convex functions have been established by Zalmai [41]. He utilized these optimality criteria as a basis for constructing two parametric and four parameter-free duality models and proving appropriate duality theorems. Osuna-Gómez et al. [33] established the optimality condition and duality theorems for a class of multiobjective fractional programs under generalized convexity assumptions by applying the parametric approach. Using the properties of sublinear functionals and generalized convex functions, Liang et al. [23] derived sufficient optimality conditions, formulated three types of duals and proved duality results for a class of nonconvex multiobjective fractional programming problems. Based on the former conclusions, by adding conditions to objective functions and constraint functions and by changing Kuhn-Tucker conditions, Zhang and Wu [43] proved the optimality conditions and duality theorems for the considered three kinds of nonlinear fractional programming problems under weaker convexity conditions. Recently, Antczak and Verma [2] proved optimality conditions and duality results for nondifferentiable multiobjective fractional programming problems under $(b, \Psi, \Phi, \rho)$-univexity hypotheses. In [12], Ho established the equivalence between the saddle point and an efficient solution of the considered multiobjective fractional problem under exponential $(p, r)$-invexity assumptions. For more information about fractional programming problems, the reader may consult the research bibliography compiled by Stancu-Minasian [35, 36].

Further, various types of generalizations of convexity theory have played an important role in the evolution of the mathematical programming. During the past decades, the generalizations of convexity were enriched with and without differentiability assumptions. One of the notions of generalized convexity introduced to weaken the convexity assumptions in proving the fundamental results in optimization theory for a new class of nonconvex (not necessarily) differentiable optimization problems is the concept of $E$-convexity which was introduced by Youness [40]. This kind of generalized convexity is based on the effect of an operator $E: R^{n} \rightarrow R^{n}$ on the sets and the domain of the definition of functions. Recently, Antczak and Abdulaleem [1] proved the so-called $E$-optimality conditions and Wolfe $E$-duality for $E$-differentiable vector optimization problems with both inequality and equality constraints.

In this paper, we consider a new class of (not necessarily differentiable) multiobjective fractional programming problems with both inequality and equality constraints in which the involved functions are $E$-differentiable. For the considered $E$-differentiable fractional programming problem, its equivalent multiobjective fractional $E$-programming problem is constructed. Then, we use the Dinkelbach parametric approach for the multiobjective fractional $E$-programming problem. Since it is equivalent to the considered $E$-differentiable multiobjective fractional programming problem, then, in fact, we use the parametric approach for solving the original extremum problem. Hence, we derive the parametric necessary $E$-optimality conditions for (weakly) $E$-efficiency of the considered $E$-differentiable multiobjective fractional programming problem. Under $E$ convexity assumptions, we also prove sufficient optimality conditions for the considered $E$-differentiable multiobjective fractional programming problem. We illustrate the $E$-optimality results established in the paper by a suitable example of an $E$-differentiable multiobjective fractional programming problem involving $E$-convex functions.

Further, we also introduce two types of vector dual models for the multiobjective fractional $E$-programming problem which, at the same time, are the so-called vector 
$E$-dual problems for the original $E$-differentiable multiobjective fractional programming problem. We establish several duality results between the multiobjective fractional $E$ programming problem and its vector duals also under appropriate $E$-convexity hypotheses which we use in proving several so-called $E$-duality theorems between the original $E$ differentiable multiobjective fractional programming problem and its vector $E$-dual problems. It turns out that in order to prove optimality and duality results for the considered class of nonsmooth multiobjective fractional programming problems are not applicable similar results established in the literature for smooth multiobjective fractional programming problems. However, we use tools for differentiable extremum problems in proving these fundamental results for such nondifferentiable vector optimization problems.

\section{Preliminaries and notations}

In this section, we provide some definitions and some results that we shall use in the sequel.

Throughout this paper the following conventions vectors $x=\left(x_{1}, x_{2}, \ldots, x_{n}\right)^{T}$ and $y=$ $\left(y_{1}, y_{2}, \ldots, y_{n}\right)^{T}$ in $R^{n}$ will be followed:

(i) $x=y$ if and only if $x_{i}=y_{i}$ for all $i=1,2, \ldots, n$;

(ii) $x>y$ if and only if $x_{i}>y_{i}$ for all $i=1,2, \ldots, n$;

(iii) $x \geqq y$ if and only if $x_{i} \geqq y_{i}$ for all $i=1,2, \ldots, n$;

(iv) $x \geq y$ if and only if $x_{i} \geqq y_{i}$ for all $i=1,2, \ldots, n$ but $x \neq y$;

(v) $x \ngtr y$ is the negation of $x>y$.

The definition of an $E$-convex set and the definition of an $E$-convex function were introduced by Youness [40]. Now, we recall them for a common reader.

Definition 1 ([40]) A set $S \subseteq R^{n}$ is said to be $E$-convex (with respect to an operator $E$ : $R^{n} \rightarrow R^{n}$ ) if and only if, the following relation

$$
E(u)+\lambda(E(x)-E(u)) \in S
$$

holds for all $x, u \in S$ and any $\lambda \in[0,1]$.

Definition 2 ([40]) Let $E: R^{n} \rightarrow R^{n}$ and $S$ be a nonempty $E$-convex subset of $R^{n}$. A realvalued function $f: R^{n} \rightarrow R$ is said to be $E$-convex on $S$ if and only if, the inequality

$$
f(\lambda E(x)+(1-\lambda) E(u)) \leqq \lambda f(E(x))+(1-\lambda) f(E(u))
$$

holds for all $x, u \in S$ and any $\lambda \in[0,1]$.

Definition 3 Let $E: R^{n} \rightarrow R^{n}$ and $S$ be a nonempty $E$-convex subset of $R^{n}$. A real-valued function $f: R^{n} \rightarrow R$ is said to be strictly $E$-convex (with respect to an operator $E: R^{n} \rightarrow R^{n}$ ) on $S$ if and only if, the inequality

$$
f(\lambda E(x)+(1-\lambda) E(u))<\lambda f(E(x))+(1-\lambda) f(E(u))
$$

holds for all $x, u \in S, E(x) \neq E(u)$, and any $\lambda \in(0,1)$. 
Definition 4 ([29]) Let $E: R^{n} \rightarrow R^{n}$ and $f: R^{n} \rightarrow R$ be a (not necessarily) differentiable function at a given point $u$. It is said that $f$ is an $E$-differentiable function at $u$ if and only if $f \circ E$ is a differentiable function at $u$ (in the usual sense), that is,

$$
(f \circ E)(x)=(f \circ E)(u)+\nabla(f \circ E)(u)(x-u)+\theta(u, x-u)\|x-u\|,
$$

where $\theta(u, x-u) \rightarrow 0$ as $x \rightarrow u$.

Proposition 5 ([1]) Let $E: R^{n} \rightarrow R^{n}, S$ be an E-convex subset of $R^{n}$ and $f: R^{n} \rightarrow R$ be an $E$ convex (strictly E-convex) function on $S$ and $u \in S$. Further, assume that $f$ is E-differentiable at $u$. Then the inequality

$$
f(E(x))-f(E(u)) \geqq \nabla f(E(u))(E(x)-E(u)) \quad(>)
$$

holds for all $x \in S,(E(x)) \neq(E(u))$.

\section{Fractional multiobjective programming and E-optimality conditions}

In the paper, we consider the multiobjective fractional programming problem defined by

$$
\begin{array}{ll}
\min \left(\frac{f_{1}(x)}{q_{1}(x)}, \ldots, \frac{f_{p}(x)}{q_{p}(x)}\right) \\
\text { subject to } \quad g_{j}(x) \leqq 0, \quad j=1, \ldots, m, \\
h_{k}(x)=0, \quad k=1, \ldots, r,
\end{array}
$$

where $f_{i}: R^{n} \rightarrow R, q_{i}: R^{n} \rightarrow R, i \in I=\{1, \ldots, p\}, g_{j}: R^{n} \rightarrow R, j \in J=\{1, \ldots, m\}, h_{k}: R^{n} \rightarrow$ $R, k \in K=\{1, \ldots, r\}$, are $E$-differentiable functions on $R^{n}$. Further, we shall assume that $f_{i}(x) \geqq 0, i \in I, q_{i}(x)>0, i \in I$, for all $x \in R^{n}$. Let $D:=\left\{x \in R^{n}: g_{j}(x) \leqq 0, j \in J, h_{k}(x)=0\right.$, $k \in K\}$ be the set of all feasible solutions in (MFP).

Let $E: R^{n} \rightarrow R^{n}$ be a given one-to-one and onto operator. Now, for the considered multiobjective fractional programming problem (MFP), we define its associated multiobjective fractional programming problem $\left(\mathrm{MFP}_{E}\right)$ as follows:

$$
\begin{aligned}
\min \left(\frac{f_{1}(E(x))}{q_{1}(E(x))}, \ldots, \frac{f_{p}(E(x))}{q_{p}(E(x))}\right) \\
\text { subject to } \quad g_{j}(E(x)) \leqq 0, \quad j=1, \ldots, m, \\
h_{k}(E(x))=0, \quad k=1, \ldots, r,
\end{aligned}
$$

where the functions $f_{i} \circ E: R^{n} \rightarrow R, q_{i} \circ E: R^{n} \rightarrow R, i=1,2, \ldots, p, g_{j} \circ E: R^{n} \rightarrow R$, $j=1,2, \ldots, m, h_{k} \circ E: R^{n} \rightarrow R, k=1,2, \ldots, r$, are differentiable real-valued functions. Let

$$
D_{E}:=\left\{x \in R^{n}:\left(g_{j} \circ E\right)(x) \leqq 0, j \in J,\left(h_{k} \circ E\right)(x)=0, k \in K\right\}
$$

be the set of all feasible solutions of $\left(\mathrm{MFP}_{E}\right)$. We call $\left(\mathrm{MFP}_{E}\right)$ the multiobjective fractional $E$-programming problem.

We now give the result established by Antczak and Abdulaleem [1] which is useful in proving the main results in the paper. 
Lemma 6 ([1]) Let $E: R^{n} \rightarrow R^{n}$ be a one-to-one and onto operator. Then $E\left(D_{E}\right)=D$.

Now, we give the definitions of optimal solutions for vector optimization problems (MFP) and $\left(\mathrm{MFP}_{E}\right)$. For such a multicriterion optimization problem as the multiobjective fractional programming problem $\left(\mathrm{MFP}_{E}\right)$, we define its optimal solutions as (weak) Pareto ((weakly) efficient) solutions and, moreover, we define optimal solutions of the original multiobjective fractional programming problems (MFP) as (weak) $E$-Pareto ((weakly) E-efficient) solutions.

Let us denote $\varphi=\left(\varphi_{1}, \ldots, \varphi_{p}\right): R^{n} \rightarrow R^{p}$, where $\varphi_{i}(z)=\frac{f_{i}(z)}{q i(z)}, i \in I$.

Definition $7 \bar{x} \in D_{E}$ is said to be a weak Pareto solution (a weakly efficient solution) of $\left(\mathrm{MFP}_{E}\right)$ if and only if there is no another $x \in D_{E}$ such that

$$
\varphi(E(x))<\varphi(E(\bar{x}))
$$

Definition $8 \bar{x} \in D_{E}$ is said to be a Pareto solution (an efficient solution) of $\left(\mathrm{MFP}_{E}\right)$ if and only if there is no another $x \in D_{E}$ such that

$$
\varphi(E(x)) \leq \varphi(E(\bar{x}))
$$

Definition $9 E(\bar{x}) \in D$ is said to be a weak $E$-Pareto solution (a weakly $E$-efficient solution) of (MFP) if and only if there is no another feasible solution $E(x) \in D$ such that (5) is satisfied.

Definition $10 E(\bar{x}) \in D$ is said to be an $E$-Pareto solution (an $E$-efficient solution) of (MFP) if and only if there is no another feasible solution $E(x) \in D$ such that (6) is satisfied.

Proposition 11 ([1]) Let $E: R^{n} \rightarrow R^{n}$ be a one-to-one and onto operator. $\bar{x} \in D_{E}$ is a weak Pareto solution (a Pareto solution) (a weakly efficient solution (an efficient solution)) of $\left(\mathrm{MFP}_{E}\right)$ if and only if $E(\bar{x}) \in D$ is a weak E-Pareto solution (an E-Pareto solution) (a weakly E-efficient solution (an E-efficient solution)) of (MFP).

Dinkelbach [9] and Jagannathan [13] introduced the parametric approach for solving scalar fractional optimization problems. Following Crouzeix et al. [7, 8] and Zalmai [41], we use the parametric approach for solving the nonlinear multiobjective fractional $E$ programming problem $\left(\mathrm{MFP}_{E}\right)$ and, thus, the considered nonlinear multiobjective fractional programming problem $\left(\mathrm{MFP}_{E}\right)$. Therefore, for the foregoing multiobjective fractional programming problems (MFP) and $\left(\mathrm{MFP}_{E}\right)$, we define their associated nonfractional parametric vector optimization problem $\left(\mathrm{MP}_{E}^{v}\right)$ for $v^{E}=\left(v_{1}^{E}, \ldots, v_{p}^{E}\right) \in R^{p}$ as follows:

$$
\begin{array}{ll}
\min \left(f_{1}(E(x))-v_{1}^{E} q_{1}(E(x)), \ldots, f_{p}(E(x))-v_{p}^{E} q_{p}(E(x))\right) & \\
\text { subject to } & g_{j}(E(x)) \leqq 0, \quad j=1, \ldots, m, \\
& h_{k}(E(x))=0, \quad k=1, \ldots, r .
\end{array}
$$

Note that the set of feasible solutions of the nonfractional parametric vector optimization problem $\left(\mathrm{MP}_{E}^{{ }^{E}}\right)$ is the same as the set of all feasible solutions of $\left(\mathrm{MFP}_{E}\right)$ 
Using the following lemmas, we can obtain the parametric Karush-Kuhn-Tucker type necessary $E$-optimality conditions for the considered (not necessarily) differentiable multiobjective fractional programming problem (MFP).

Lemma $12 \bar{x}$ is a weakly efficient solution (an efficient solution) of the multiobjective fractional E-programming problem $\left(\mathrm{MFP}_{E}\right.$ ) if and only if $\bar{x}$ is a weakly efficient solution (an efficient solution) of the nonfractional parametric vector optimization problem $\left(\mathrm{MP}_{E}^{\bar{\nu}^{E}}\right)$, where $\bar{v}_{i}^{E}=\frac{f_{i}(\bar{x})}{q_{i}(\bar{x})}, i \in I$.

The following result follows directly from Proposition 11.

Lemma $13 E(\bar{x})$ is a weakly E-efficient solution (an E-efficient solution) of the considered multiobjective fractional programming problem (MFP) if and only if $\bar{x}$ is a weakly efficient solution (an efficient solution) of the nonfractional parametric vector optimization problem $\left(\mathrm{MP}_{E}^{\bar{v}^{E}}\right)$, where $\bar{v}_{i}^{E}=\frac{f_{i}(\bar{x})}{q_{i}(\bar{x})}, i \in I$.

Subsequently, necessary optimality conditions similar to the well-known KarushKuhn-Tucker necessary optimality conditions for a smooth nonlinear programming problem were presented for various differentiable multiobjective fractional programming problems (for example, see [14, 22, 23, 28, 33]). One of such optimality criteria are the following parametric Karush-Kuhn-Tucker optimality conditions, which are necessary for optimality of a feasible solution $\bar{x}$ in the problem $\left(\mathrm{MP}_{E}^{v^{E}}\right)$. In order to prove the so-called parametric necessary optimality conditions, it can be utilized the equivalence between the problems (MFP) and $\left(\mathrm{MP}_{E}^{\nu^{E}}\right)$ (see Proposition 11).

Theorem 14 (Parametric necessary optimality conditions for $\left(\mathrm{MFP}_{E}\right)$ ) Let $\bar{x} \in D_{E}$ be a weakly efficient solution of the multiobjective fractional E-programming problem $\left(\mathrm{MFP}_{E}\right)$. Further, assume that the so-called E-Abadie constraint qualification $\left(\mathrm{ACQ}_{E}\right)$ [1] is satisfied at $\bar{x}$. Then there exist $\bar{\lambda} \in R^{p}, \bar{\mu} \in R^{m}, \bar{\xi} \in R^{r}$ and $\bar{v}^{E} \in R^{p}$ such that the following conditions are satisfied:

$$
\begin{aligned}
& \sum_{i=1}^{p} \bar{\lambda}_{i}\left(\nabla f_{i}(E(\bar{x}))-\bar{v}_{i}^{E} \nabla q_{i}(E(\bar{x}))\right)+\sum_{j=1}^{m} \bar{\mu}_{j} \nabla g_{j}(E(\bar{x}))+\sum_{k=1}^{r} \bar{\xi}_{k} \nabla h_{k}(E(\bar{x}))=0, \\
& \bar{\mu}_{j} g_{j}(E(\bar{x}))=0, \quad j=1, \ldots, m, \\
& \bar{\lambda}_{i} \geqq 0, \quad i \in I, \quad \sum_{i=1}^{p} \bar{\lambda}_{i}=1, \quad \bar{\mu}_{j} \geqq 0, \quad j \in J .
\end{aligned}
$$

Proof Since $\bar{x} \in D_{E}$ is a weakly efficient solution of the multiobjective fractional $E$ programming problem $\left(\mathrm{MFP}_{E}\right)$, by Lemma $12, \bar{x} \in D_{E}$ is also a weakly efficient solution of its associated nonfractional parametric vector optimization problem $\left(\mathrm{MP}_{E}^{v}\right)$. Note that all hypotheses of Theorem 29 [1] are fulfilled. Then there exist Lagrange multipliers $\bar{\lambda} \in R^{p}$, $\bar{\mu} \in R^{m}$ and $\bar{\xi} \in R^{q}$ such that

$$
\sum_{i=1}^{p} \frac{\lambda_{i}^{*}}{q_{i}(E(\bar{x}))}\left[\nabla f_{i}(E(\bar{x}))-\bar{v}_{i}^{E} \nabla q_{i}(E(\bar{x}))\right]+\sum_{j=1}^{m} \mu_{j}^{*} \nabla g_{j}(E(\bar{x}))+\sum_{k=1}^{r} \xi_{k}^{*} \nabla h_{k}(E(\bar{x}))=0
$$




$$
\begin{aligned}
& \mu_{j}^{*}\left(g_{j} \circ E\right)(\bar{x})=0, \quad j \in J, \\
& \lambda^{*} \geq 0, \quad \mu^{*} \geqq 0,
\end{aligned}
$$

where $\bar{v}_{i}^{E}=\frac{f_{i}(E(\bar{x}))}{q_{i}(E(\bar{x}))}, i \in I$. Now, let us denote $\bar{\lambda}_{i}=\frac{\frac{\lambda_{i}^{*}}{q_{i}(E(\bar{x}))}}{\sum_{i=1}^{p} \frac{\lambda_{i}^{*}}{q_{i}(E(\bar{x}))}}, i \in I, \bar{\mu}_{j}=\frac{\mu_{j}^{*}}{\sum_{i=1}^{p} \frac{\lambda_{i}^{*}}{q_{i}(E(\bar{x}))}}, j \in J$, $\bar{\xi}_{k}=\frac{\xi_{k}^{*}}{\sum_{i=1}^{p} \frac{\lambda_{i}^{*}}{q_{i}(E(\bar{x}))}}, k \in K$. Note that $\bar{\lambda}=\left(\bar{\lambda}_{1}, \ldots, \bar{\lambda}_{p}\right) \geq 0, \sum_{i=1}^{p} \bar{\lambda}_{i}=1, \bar{\mu}=\left(\bar{\mu}_{1}, \ldots, \bar{\mu}_{m}\right) \geq 0$ and, moreover, the necessary optimality conditions (7)-(9) are satisfied at $\bar{x}$ with Lagrange multipliers $\bar{\lambda} \in R^{p}, \bar{\mu} \in R^{m}$ and $\bar{\xi} \in R^{r}$, where $\bar{v}_{i}^{E}=\frac{f_{i}(E(\bar{x}))}{q_{i}(E(\bar{x}))}, i \in I$. This completes the proof of this theorem.

Theorem 15 (Parametric necessary E-optimality conditions for (MFP)) Let $E(\bar{x})$ be a weakly E-efficient solution of the considered multiobjective fractional programming problem (MFP). Further, assume that the suitable constraint qualification is satisfied at $\bar{x}$. Then there exist $\bar{\lambda} \in R^{p}, \bar{\mu} \in R^{m}, \bar{\xi} \in R^{r}$ and $\bar{v}^{E} \in R^{p}$ such that the conditions (7)-(9) are satisfied.

Remark 16 The conditions (7)-(9) are the parametric Karush-Kuhn-Tucker necessary optimality conditions for the multiobjective fractional $E$-programming problem $\left(\mathrm{MFP}_{E}\right)$. Thus, they are also the necessary $E$-optimality conditions for the original multiobjective fractional programming problem (MFP). Therefore, we call them the parametric $E$ Karush-Kuhn-Tucker necessary optimality conditions for the considered $E$-differentiable multiobjective fractional programming problem (MFP). Note that, although the functions involved in the multiobjective fractional programming problem (MFP) are not necessarily differentiable at a weak Pareto solution $\bar{x}$ (since they are assumed to be $E$-differentiable only), we formulate the $E$-Karush-Kuhn-Tucker necessary optimality conditions for such a nonsmooth extremum problem by using tools for differentiable optimization problems.

Now, under $E$-convexity hypotheses, we prove the sufficient $E$-optimality conditions for the considered multiobjective fractional programming problem (MFP). First, we prove the sufficient optimality conditions for the multiobjective fractional $E$-programming problem $\left(\mathrm{MFP}_{E}\right)$ and we use them in proving the foregoing sufficient conditions for the original $E$ differentiable multiobjective fractional programming problem (MFP).

Theorem 17 (Sufficient optimality conditions for $\left(\mathrm{MFP}_{E}\right)$ ) Let $\bar{x}$ be a feasible solution of the multiobjective fractional E-programming problem $\left(\mathrm{MFP}_{E}\right), \bar{v}_{i}^{E}=\frac{f_{i}(E(\bar{x}))}{q_{i}(E(\bar{x}))}, i \in I$, and the necessary optimality conditions (7)-(9) be satisfied at $\bar{x}$ with the Lagrange multipliers $\bar{\lambda} \in R^{p}, \bar{\mu} \in R^{m}$ and $\bar{\xi} \in R^{r}$. Further, assume that the following hypotheses are fulfilled:

(a) each function $f_{i}, i \in I$, is (strictly) E-convex at $\bar{x}$ on $D_{E}$,

(b) each function $-q_{i}, i \in I$, is E-convex at $\bar{x}$ on $D_{E}$,

(c) each function $g_{j}, j \in J_{E}(\bar{x})$, is E-convex at $\bar{x}$ on $D_{E}$,

(d) each function $h_{k}, k \in K_{E}^{+}(\bar{x}):=\{k \in K: \bar{\xi}>0\}$, is E-convex at $\bar{x}$ on $D_{E}$,

(e) each function $-h_{k}, k \in K_{E}^{-}(\bar{x}):=\{k \in K: \bar{\xi}<0\}$, is E-convex at $\bar{x}$ on $D_{E}$.

Then $\bar{x}$ is a weakly efficient solution (an efficient solution) of the multiobjective fractional E-programming problem $\left(\mathrm{MFP}_{E}\right)$ and, at the same time, $E(\bar{x})$ is a weakly E-efficient solution (an E-efficient solution) of the original E-differentiable multiobjective fractional programming problem (MFP). 
Proof Let the necessary optimality conditions (7)-(9) be satisfied at $\bar{x} \in D_{E}$ with Lagrange multipliers $\bar{\lambda} \in R^{p}, \bar{\mu} \in R^{m}$ and $\bar{\xi} \in R^{r}$. Suppose, contrary to the result, that $\bar{x}$ is not an efficient solution of the problem $\left(\mathrm{MFP}_{E}\right)$. Hence, by Definition 7 , there exists $\widetilde{x} \in D_{E}$ such that

$$
\begin{aligned}
& \frac{f_{i}(E(\widetilde{x}))}{q_{i}(E(\widetilde{x}))} \leqq \frac{f_{i}(E(\bar{x}))}{q_{i}(E(\bar{x}))}, \quad i \in I, \\
& \frac{f_{i^{*}}(E(\widetilde{x}))}{q_{i^{*}}(E(\widetilde{x}))}<\frac{f_{i^{*}}(E(\bar{x}))}{q_{i^{*}}(E(\bar{x}))} \quad \text { for some } i^{*} \in I .
\end{aligned}
$$

Hence, the inequalities above imply

$$
\begin{aligned}
& f_{i}(E(\widetilde{x}))-\bar{v}_{i}^{E} q_{i}(E(\widetilde{x})) \leqq f_{i}(E(\bar{x}))-\bar{v}_{i} q_{i}(E(\bar{x})), \quad i \in I, \\
& f_{i^{*}}(E(\widetilde{x}))-\bar{v}_{i^{*}}^{E} q_{i^{*}}(E(\widetilde{x}))<f_{i^{*}}(E(\bar{x}))-\bar{v}_{i^{*}} q_{i^{*}}(E(\bar{x})) \quad \text { for some } i^{*} \in I .
\end{aligned}
$$

Adding both sides of the inequalities (13) and (14), we get

$$
\sum_{i=1}^{p} \bar{\lambda}_{i}\left[f_{i}(E(\widetilde{x}))-\bar{v}_{i}^{E} q_{i}(E(\widetilde{x}))\right]<\sum_{i=1}^{p} \bar{\lambda}_{i}\left[f_{i}(E(\bar{x}))-\bar{v}_{i}^{E} q_{i}(E(\bar{x}))\right] .
$$

Using the assumptions (a) and (b), we see, by Proposition 5, that the inequalities

$$
\begin{aligned}
& f_{i}(E(x))-f_{i}(E(\bar{x}))>\nabla f_{i}(E(\bar{x}))(E(x)-E(\bar{x})), \quad i \in I, \\
& -q_{i}(E(x))+q_{i}(E(\bar{x})) \geqq-\nabla q_{i}(E(\bar{x}))(E(x)-E(\bar{x})), \quad i \in I, \\
& g_{j}(E(x))-g_{j}(E(\bar{x})) \geqq \nabla g_{j}(E(\bar{x}))(E(x)-E(\bar{x})), \quad j \in J(\bar{x}), \\
& h_{k}(E(x))-h_{k}(E(\bar{x})) \geqq \nabla h_{k}(E(\bar{x}))(E(x)-E(\bar{x})), \quad k \in K_{E}^{+}(\bar{x}), \\
& -h_{k}(E(x))+h_{k}(E(\bar{x})) \geqq-\nabla h_{k}(E(\bar{x}))(E(x)-E(\bar{x})), \quad k \in K_{E}^{-}(\bar{x})
\end{aligned}
$$

hold for all $x \in D_{E}$. Therefore, they are also satisfied for $x=\tilde{x}$. Thus,

$$
\begin{aligned}
& f_{i}(E(\widetilde{x}))-f_{i}(E(\bar{x}))>\nabla f_{i}(E(\bar{x}))(E(\widetilde{x})-E(\bar{x})), \quad i \in I, \\
& -q_{i}(E(\widetilde{x}))+q_{i}(E(\bar{x})) \geqq-\nabla q_{i}(E(\bar{x}))(E(\widetilde{x})-E(\bar{x})), \quad i \in I, \\
& g_{j}(E(\widetilde{x}))-g_{j}(E(\bar{x})) \geqq \nabla g_{j}(E(\bar{x}))(E(\widetilde{x})-E(\bar{x})), \quad j \in J_{E}(\bar{x}), \\
& h_{k}(E(\widetilde{x}))-h_{k}(E(\bar{x})) \geqq \nabla h_{k}(E(\bar{x}))(E(\widetilde{x})-E(\bar{x})), \quad k \in K_{E}^{+}(\bar{x}), \\
& -h_{k}(E(\widetilde{x}))+h_{k}(E(\bar{x})) \geqq-\nabla h_{k}(E(\bar{x}))(E(\widetilde{x})-E(\bar{x})), \quad k \in K_{E}^{-}(\bar{x}) .
\end{aligned}
$$

We multiply the inequalities above by the corresponding Lagrange multipliers and, moreover, we multiply (22) extra by $\bar{v}_{i}^{E}=\frac{f_{i}(E(\bar{x}))}{q_{i}(E(\bar{x}))} \geqq 0, i \in I$. After summing the resulting inequal- 
ities and taking into account the Lagrange multipliers equal to 0, (21)-(25) yield

$$
\begin{aligned}
\sum_{i=1}^{p} & \bar{\lambda}_{i}\left[f_{i}(E(\widetilde{x}))-\bar{v}_{i}^{E} q_{i}(E(\widetilde{x}))\right]+\sum_{j=1}^{m} \bar{\mu}_{j} g_{j}(E(\widetilde{x}))+\sum_{k=1}^{r} \xi_{k} h_{k}(E(\widetilde{x})) \\
& -\left\{\sum_{i=1}^{p} \bar{\lambda}_{i}\left[f_{i}(E(\bar{x}))-\bar{v}_{i}^{E} q_{i}(E(\bar{x}))\right]+\sum_{j=1}^{m} \bar{\mu}_{j} g_{j}(E(\bar{x}))+\sum_{k=1}^{r} \xi_{k} h_{k}(E(\bar{x}))\right\} \\
\geqq & {\left[\sum_{i=1}^{p} \bar{\lambda}_{i} \nabla f_{i}(E(\bar{x}))-\bar{v}_{i}^{E} \nabla q_{i}(E(\bar{x}))+\sum_{j=1}^{m} \bar{\mu}_{j} \nabla g_{j}(E(\bar{x}))\right.} \\
& \left.+\sum_{k=1}^{r} \xi_{k} \nabla h_{k}(E(\bar{x}))\right](E(\widetilde{x})-E(\bar{x})) .
\end{aligned}
$$

Hence, by the parametric necessary optimality condition (7), (26) implies

$$
\begin{aligned}
& \sum_{i=1}^{p} \bar{\lambda}_{i}\left[f_{i}(E(\widetilde{x}))-\bar{v}_{i}^{E} q_{i}(E(\widetilde{x}))\right]+\sum_{j=1}^{m} \bar{\mu}_{j} g_{j}(E(\widetilde{x}))+\sum_{k=1}^{r} \xi_{k} h_{k}(E(\widetilde{x})) \\
& \quad \geqq \sum_{i=1}^{p} \bar{\lambda}_{i}\left[f_{i}(E(\bar{x}))-\bar{v}_{i}^{E} q_{i}(E(\bar{x}))\right]+\sum_{j=1}^{m} \bar{\mu}_{j} g_{j}(E(\bar{x}))+\sum_{k=1}^{r} \xi_{k} h_{k}(E(\bar{x})) .
\end{aligned}
$$

Using the parametric necessary optimality condition (8) together with the feasibility of $\widetilde{x}$ and $\bar{x}$ in the problem $\left(\mathrm{MFP}_{E}\right)$, we see that the inequality

$$
\sum_{i=1}^{p} \bar{\lambda}_{i}\left[f_{i}(E(\widetilde{x}))-\bar{v}_{i}^{E} q_{i}(E(\widetilde{x}))\right] \geqq \sum_{i=1}^{p} \bar{\lambda}_{i}\left[f_{i}(E(\bar{x}))-\bar{v}_{i}^{E} q_{i}(E(\bar{x}))\right]
$$

holds, contradicting (15). The proof in the case of weakly $E$-efficiency is similar and, therefore, it has been omitted in the paper. Thus, the proof of this theorem is completed.

Now, we give an example of a multiobjective fractional programming problem involving $E$-differentiable functions. In order to prove $E$-efficiency of a feasible solution $E(\bar{x})$ in such a nondifferentiable extremum problem, the concept of $E$-convexity may be applied.

Example 18 Consider the following nondifferentiable multiobjective fractional programming problem:

$$
\begin{gathered}
\min \varphi\left(x_{1}, x_{2}\right)=\left(\frac{3 x_{1}^{2}-2 x_{2}^{\frac{2}{3}}+4 x_{1}+8 x_{2}^{\frac{1}{3}}+12}{x_{1}^{2}-x_{2}^{\frac{2}{3}}-8 x_{2}^{\frac{1}{3}}+12}, \frac{3 x_{1}^{2}-2 x_{2}^{\frac{2}{3}}+4 x_{1}+8 x_{2}^{\frac{1}{3}}+9}{x_{1}^{2}-x_{2}^{\frac{2}{3}}-8 x_{2}^{\frac{1}{3}}+9}\right) \\
\text { subject to } g\left(x_{1}, x_{2}\right)=x_{2}^{\frac{1}{3}}-x_{1} \leqq 0 \\
h\left(x_{1}, x_{2}\right)=x_{1}^{2}-x_{2}^{\frac{1}{3}}=0 .
\end{gathered}
$$

Note that $D=\left\{\left(x_{1}, x_{2}\right) \in R^{2}: x_{2}^{\frac{1}{3}}-x_{1} \leqq 0 \wedge x_{1}^{2}-x_{2}^{\frac{1}{3}}=0\right\}$. Let $E: R^{2} \rightarrow R^{2}$ be an one-to-one and onto mapping defined by $E\left(x_{1}, x_{2}\right)=\left(x_{1}, x_{2}^{3}\right)$. Now, for the considered nondifferentiable 
multiobjective fractional programming problem (MFP1), we define its associated multiobjective fractional programming problem $\left(M F P 1_{E}\right)$ as follows:

$$
\begin{array}{cl}
\min \varphi\left(E\left(x_{1}, x_{2}\right)\right)=\left(\frac{3 x_{1}^{2}-2 x_{2}^{2}+4 x_{1}+8 x_{2}+12}{x_{1}^{2}-x_{2}^{2}-8 x_{2}+12}, \frac{3 x_{1}^{2}-2 x_{2}^{2}+4 x_{1}+8 x_{2}+9}{x_{1}^{2}-x_{2}^{2}-8 x_{2}+9}\right) \\
\text { subject to } \quad g\left(E\left(x_{1}, x_{2}\right)\right)=x_{2}-x_{1} \leqq 0, \\
h\left(E\left(x_{1}, x_{2}\right)\right)=x_{1}^{2}-x_{2}=0 .
\end{array}
$$

Note that $D_{E}=\left\{\left(x_{1}, x_{2}\right) \in R^{2}: x_{2}=x_{1}^{2} \wedge 0 \leqq x_{1} \leqq 1\right\}$ and $\bar{x}=(0,0)$ is a feasible solution of the multiobjective fractional programming problem $\left(M F P 1_{E}\right)$. Further, note that all functions constituting the considered multiobjective fractional programming problem (MFP1) are $E$-differentiable at $\bar{x}=(0,0)$. Then it can also be shown that the parametric necessary optimality conditions (7)-(9) are fulfilled at $\bar{x}=(0,0)$ with Lagrange multipliers $\bar{\lambda}_{1}=\frac{1}{4}$, $\bar{\lambda}_{2}=\frac{1}{4}, \bar{\mu}=2$ and $\bar{\xi}=10$. Further, it can be proved that $f,-q, g$, and $h$ are $E$-convex at $\bar{x}$ on $D_{E}$. Since all hypotheses of Theorem 17 are fulfilled, it is possible to use the sufficient conditions formulated in this theorem to show that $\bar{x}=(0,0)$ is an efficient solution of the problem $\left(\mathrm{MFP}_{E}\right)$ and, thus, $E(\bar{x})$ is also an $E$-efficient solution of the problem (MFP1).

Remark 19 Note that it is not possible to use several optimality conditions from the literature to the multiobjective fractional programming problem (MFP1) considered in Example 18. Indeed, all optimality conditions for smooth multiobjective fractional programming problems (see, for example, [15, 21-23, 31, 33, 38, 43]) are not applicable for (MFP1), due to the fact that the problem (MFP1) is nondifferentiable. Also sufficient optimality conditions established under convexity and many generalized convexity assumptions for nondifferentiable multiobjective fractional programming problems are not useful for (MFP1) (see, for example, [4-6, 24, 37]). This is a consequence of the fact that the functions constituting the multiobjective fractional programming problem (MFP1) are not convex and, in general, it can be difficult to show that they belong to suitable classes of generalized convex functions as it requires in sufficient optimality conditions even from the works mentioned above. However, since the optimality conditions established in this paper are applicable for the problem (MFP1), this means that they can be used successfully in finding optimality solutions (that is, (weakly) $E$-efficient solutions) of $E$-differentiable multiobjective fractional programming problems, that is, for a new class of (not necessarily differentiable) multiobjective fractional programming problems which have not been considered in the literature so far.

\section{Schaible $E$-duality}

In this section, we define the vector dual problem $\left(\mathrm{MSD}_{E}\right)$ in the sense of Schaible [34] for the multiobjective fractional $E$-programming problem $\left(\mathrm{MFP}_{E}\right)$ and we prove several Schaible duality results between $\left(\mathrm{MFP}_{E}\right)$ and $\left(\mathrm{MSD}_{E}\right)$ under appropriate $E$-convexity hypotheses. Thus, we formulate the vector $E$-dual problem $\left(\mathrm{MSD}_{E}\right)$ in the sense of Schaible [34] for the considered multiobjective fractional programming problem (MFP). Further, we use Schaible duality results established between $\left(\mathrm{MFP}_{E}\right)$ and $\left(\mathrm{MSD}_{E}\right)$ in proving the so-called $E$-duality results in the sense of Schaible between (MFP) and $\left(\mathrm{MSD}_{E}\right)$.

Let $E: R^{n} \rightarrow R^{n}$ be a given one-to-one and onto operator. Now, we define the parametric vector dual problem in the sense of Schaible [34] for $\left(\mathrm{MFP}_{E}\right)$ (at the same time, the 
parametric vector E-dual problem in the sense of Schaible [34] for (MFP)) as follows:

$$
\begin{array}{ll}
\max v^{E}=\left(v_{1}^{E}, \ldots, v_{p}^{E}\right) & \\
\text { subject to } & \sum_{i=1}^{p} \lambda_{i}\left(\nabla f_{i}(E(y))-v_{i}^{E} \nabla q_{i}(E(y))\right) \\
& +\sum_{j=1}^{m} \mu_{j} \nabla g_{j}(E(y))+\sum_{k=1}^{r} \xi_{k} \nabla h_{k}(E(y))=0, \\
& f_{i}(y)-v_{i}^{E} q_{i}(y) \geqq 0, \quad i=1, \ldots, p, \\
& \sum_{j=1}^{m} \mu_{j} g_{j}(y)+\sum_{k=1}^{r} \xi_{k} h_{k}(y) \geqq 0, \\
& y \in R^{n}, \quad v^{E} \in R_{+}^{p}, \quad \lambda \geq 0, \quad \sum_{i=1}^{k} \lambda_{i}=1, \quad \mu \in R_{+}^{m}, \quad \xi \in R^{r} .
\end{array}
$$

Let $\Omega_{\mathrm{MSD}_{E}}$ denote the set of all feasible solutions of $\left(\mathrm{MSD}_{E}\right)$ and $\mathrm{pr}_{R^{n}} \Omega_{\mathrm{MSD}}$ the projection of the set $\Omega_{\mathrm{MSD}_{E}}$ on $R^{n}$, that is, $\operatorname{pr}_{R^{n}} \Omega_{\mathrm{MSD}_{E}}=\left\{y \in R^{n}: \exists v^{E} \in R_{+}^{p}, \lambda \in R_{+}^{p}, \mu \in R_{+}^{m}, \xi \in\right.$ $R^{r}$ such that $\left.\left(y, v^{E}, \lambda, \mu, \xi\right) \in \Omega_{\mathrm{MSD}_{E}}\right\}$. Let us denote $Y_{\mathrm{MSD}_{E}}=\operatorname{pr}_{R^{n}} \Omega_{\mathrm{MSD}_{E}}$.

Theorem 20 (Schaible weak duality for $\left(\mathrm{MFP}_{E}\right)$ and $\left.\left(\mathrm{MSD}_{E}\right)\right)$ Let $x$ and $\left(y, v^{E}, \lambda, \mu, \xi\right)$ be feasible solutions of the problems $\left(\mathrm{MFP}_{E}\right)$ and $\left(\mathrm{MSD}_{E}\right)$, respectively. Further, assume that:

(a) each function $f_{i}, i \in I$, is E-convex at $y$ on $D_{E} \cup Y_{\mathrm{MSD}_{E}}$,

(b) each function $-q_{i}, i \in I$, is E-convex at $y$ on $D_{E} \cup Y_{\mathrm{MSD}_{E}}$,

(c) each function $g_{j}, j \in J_{E}(y)$, is E-convex at y on $D_{E} \cup Y_{\mathrm{MSD}_{E}}$,

(d) each function $h_{k}, k \in K_{E}^{+}(y)$, is E-convex at $y$ on $D_{E} \cup Y_{\mathrm{MSD}_{E}}$,

(e) each function $-h_{k}, k \in K_{E}^{-}(y)$, is E-convex at $y$ on $D_{E} \cup Y_{\mathrm{MSD}_{E}}$.

Then $\frac{f(E(x))}{q(E(x))} \nless v^{E}$.

Proof Let $x$ and $\left(y, v^{E}, \lambda, \mu, \xi\right)$ be feasible solutions in the problems $\left(\mathrm{MFP}_{E}\right)$ and $\left(\mathrm{MSD}_{E}\right)$, respectively. We proceed by contradiction. Suppose, contrary to the result, that

$$
\frac{f(x)}{q(x)}<v^{E}
$$

Thus,

$$
\frac{f_{i}(x)}{q_{i}(x)}<v_{i}^{E}, \quad i=1, \ldots, p
$$

From $q_{i}(x)>0, i=1, \ldots, p$, it follows that

$$
f_{i}(x)-v_{i} q_{i}(x)<0, \quad i=1, \ldots, p
$$

Hence, the second constraint of $\left(\mathrm{MSD}_{E}\right)$ gives

$$
f_{i}(x)-v_{i}^{E} q_{i}(x)<f_{i}(y)-v_{i}^{E} q_{i}(y), \quad i=1, \ldots, p .
$$


By hypotheses (a)-(e), Proposition 5 implies that the inequalities

$$
\begin{aligned}
& f_{i}(E(x))-f_{i}(E(y)) \geqq \nabla f_{i}(E(y))(E(x)-E(y)), \quad i \in I, \\
& -q_{i}(E(x))+q_{i}(E(y)) \geqq-\nabla q_{i}(E(y))(E(x)-E(y)), \quad i \in I, \\
& g_{j}(E(x))-g_{j}(E(y)) \geqq \nabla g_{j}(E(y))(E(x)-E(y)), \quad j \in J_{E}(y), \\
& h_{k}(E(x))-h_{k}(E(y)) \geqq \nabla h_{k}(E(y))(E(x)-E(y)), \quad k \in K_{E}^{+}(y), \\
& -h_{k}(E(x))+h_{k}(E(y)) \geqq-\nabla h_{k}(E(y))(E(x)-E(y)), \quad k \in K_{E}^{-}(y)
\end{aligned}
$$

hold. Multiplying each inequality (33) by $v_{i}^{E} \geqq 0, i \in I$, then (31)-(33) yield

$$
\left[\nabla f_{i}(E(y))-v_{i}^{E} \nabla q_{i}(E(y))\right](E(x)-E(y))<0, \quad i \in I
$$

Multiplying each inequality (34)-(37) by the corresponding Lagrange multiplier, taking into account the Lagrange multipliers equal to 0 , and then adding both sides of the resulting inequalities, we get

$$
\begin{aligned}
& \sum_{j=1}^{m} \mu_{j} g_{j}(x)+\sum_{k=1}^{r} \xi_{k} h_{k}(x)-\left[\sum_{j=1}^{m} \mu_{j} g_{j}(y)+\sum_{k=1}^{r} \xi_{k} h_{k}(y)\right] \\
& \geqq {\left[\sum_{i=1}^{p} \lambda_{i}\left[\nabla f_{i}(E(y))-v_{i}^{E} \nabla q_{i}(E(y))\right]\right.} \\
&\left.+\sum_{j=1}^{m} \mu_{j} \nabla g_{j}(E(y))+\sum_{k=1}^{r} \xi_{k} \nabla h_{k}(E(y))\right](E(x)-E(y)) .
\end{aligned}
$$

From $x \in D_{E}$ and $\left(y, v^{E}, \lambda, \mu, \xi\right) \in \Omega_{\mathrm{MSD}_{E}}$, it follows that the inequality

$$
\begin{aligned}
& {\left[\sum_{i=1}^{p} \lambda_{i}\left[\nabla f_{i}(E(y))-v_{i}^{E} \nabla q_{i}(E(y))\right]\right.} \\
& \left.\quad+\sum_{j=1}^{m} \mu_{j} \nabla g_{j}(E(y))+\sum_{k=1}^{r} \xi_{k} \nabla h_{k}(E(y))\right](E(x)-E(y))<0
\end{aligned}
$$

holds, contradicting (27). This completes the proof of this theorem.

Theorem 21 (Schaible weak E-duality between (MFP) and $\left(\mathrm{MSD}_{E}\right)$ ) Let $E(x)$ and $\left(y, v^{E}, \lambda, \mu, \xi\right)$ be any feasible solutions of the problems $\left(\mathrm{MFP}_{E}\right)$ and $\left(\mathrm{MSD}_{E}\right)$, respectively. Further, assume that all hypotheses of Theorem 20 are fulfilled. Then $\frac{f(E(x))}{q(E(x))} \nless v^{E}$.

Proof The proof of this theorem follows directly from Theorem 20 and Proposition 11.

If we impose some stronger $E$-convexity assumption on the objective function $f$, then the following result is true.

Theorem 22 (Schaible weak duality for $\left(\mathrm{MFP}_{E}\right)$ and $\left.\left(\mathrm{MSD}_{E}\right)\right)$ Let $x$ and $\left(y, v^{E}, \lambda, \mu, \xi\right)$ be feasible solutions of the problems $\left(\mathrm{MFP}_{E}\right)$ and $\left(\mathrm{MSD}_{E}\right)$, respectively. Further, assume that: 
(a) each function $f_{i}, i \in I$, is strictly E-convex at y on $D_{E} \cup Y_{\mathrm{MSD}_{E}}$,

(b) each function $-q_{i}, i \in I$, is E-convex at $y$ on $D_{E} \cup Y_{\mathrm{MSD}_{E}}$,

(c) each function $g_{j}, j \in J_{E}(y)$, is $E$-convex at $y$ on $D_{E} \cup Y_{\mathrm{MSD}_{E}}$,

(d) each function $h_{k}, k \in K_{E}^{+}(y)$, is E-convex at y on $D_{E} \cup Y_{\mathrm{MSD}_{E}}$,

(e) each function $-h_{k}, k \in K_{E}^{-}(y)$, is E-convex at $y$ on $D_{E} \cup Y_{\mathrm{MSD}_{E}}$.

Then $\frac{f(E(x))}{q(E(x))} \not \leq v^{E}$.

Theorem 23 (Schaible weak $E$-duality between (MFP) and $\left(\mathrm{MSD}_{E}\right)$ ) Let $E(x)$ and $\left(y, v^{E}, \lambda, \mu, \xi\right)$ be any feasible solutions of the problems $\left(\mathrm{MFP}_{E}\right)$ and $\left(\mathrm{MSD}_{E}\right)$, respectively. Further, assume that all hypotheses of Theorem 22 are fulfilled. Then $\frac{f(E(x))}{q(E(x))} \not \leq v^{E}$.

Theorem 24 (Schaible strong duality for $\left(\mathrm{MFP}_{E}\right)$ and $\left.\left(\mathrm{MSD}_{E}\right)\right)$ Let $\bar{x}$ be a weak Pareto solution (a Pareto solution) of the multiobjective fractional E-programming problem $\left(\mathrm{MFP}_{E}\right)$ and the constraint qualification [1] be satisfied at $\bar{x}$. Then there exist $\bar{\lambda} \in R_{+}^{p}, \bar{\mu} \in R_{+}^{m}, \bar{\xi} \in R^{r}$ and $\bar{v}^{E} \in R_{+}^{p}$ such that $\left(\bar{x}, \bar{v}^{E}, \bar{\lambda}, \bar{\mu}, \bar{\xi}\right)$ is feasible in the Schaible dual problem $\left(\mathrm{MSD}_{E}\right)$ and

$$
\frac{f(E(\bar{x}))}{q(E(\bar{x}))}=\bar{v}^{E}
$$

If also all hypotheses of the weak duality theorem-Theorem 20 (Theorem 22)-are satisfied, then $\left(\bar{x}, \bar{v}^{E}, \bar{\lambda}, \bar{\mu}, \bar{\xi}\right)$ is a weakly efficient solution (an efficient solution) of a maximum type for the problem $\left(\mathrm{MSD}_{E}\right)$.

Proof By assumption, $\bar{x}$ is a weak Pareto solution (a Pareto solution) of the problem $\left(\mathrm{MFP}_{E}\right)$ and the suitable constraint qualification [1] is satisfied at $\bar{x}$. Then, by $\bar{x} \in D_{E}$ and Theorem 14, we conclude that $\left(\bar{x}, \bar{v}^{E}, \bar{\lambda}, \bar{\mu}, \bar{\xi}\right) \in \Omega_{\mathrm{MSD}_{E}}$. Obviously, the corresponding objective function value in $\left(\mathrm{MSD}_{E}\right)$ is equal to $\frac{f(E(\bar{x}))}{q(E(\bar{x}))}$. Let $\left(y, v^{E}, \lambda, \mu, \xi\right)$ be any feasible solution of $\left(\mathrm{MSD}_{E}\right)$. Then, by the weak duality theorem-Theorem 20 (Theorem 22), it follows that the inequality $\bar{v}^{E} \nless v^{E}\left(\bar{v}^{E} \nless v^{E}\right)$ holds. This implies that $\left(\bar{x}, \bar{v}^{E}, \bar{\lambda}, \bar{\mu}, \bar{\xi}\right)$ is a weakly efficient solution (an efficient solution) of a maximum type of the problem $\left(\mathrm{MSD}_{E}\right)$. This completes the proof of this theorem.

Theorem 25 (Schaible strong $E$-duality between (MFP) and $\left.\left(\mathrm{MSD}_{E}\right)\right)$ Let $E(\bar{x})$ be a weak E-Pareto solution (an E-Pareto solution) of the E-differentiable multiobjective fractional programming problem (MFP) and the E-constraint qualification [1] be satisfied. Then there exist $\bar{\lambda} \in R_{+}^{p}, \bar{\mu} \in R_{+}^{m}, \bar{\xi} \in R^{r}$ and $\bar{v}^{E} \in R_{+}^{p}$ such that $\left(\bar{x}, \bar{v}^{E}, \bar{\lambda}, \bar{\mu}, \bar{\xi}\right)$ is feasible in the Schaible dual problem $\left(\mathrm{MSD}_{E}\right)$ and

$$
\frac{f(E(\bar{x}))}{q(E(\bar{x}))}=\bar{v}^{E} .
$$

If also all hypotheses of the weak E-duality theorem-Theorem 21 (Theorem 23)-are satisfied, then $\left(\bar{x}, \bar{v}^{E}, \bar{\lambda}, \bar{\mu}, \bar{\xi}\right)$ is a weakly E-efficient solution (an E-efficient solution) of a maximum type for the problem (MSD).

Theorem 26 (Schaible converse duality between $\left(\mathrm{MFP}_{E}\right)$ and $\left.\left(\mathrm{MSD}_{E}\right)\right) \operatorname{Let}\left(\bar{y}, \bar{v}^{E}, \bar{\lambda}, \bar{\mu}, \bar{\xi}\right) \in$ $\Omega_{\mathrm{MSD}_{E}}$ be a weakly efficient solution (an efficient solution) of a maximum type in the problem (MSD) and $\bar{y} \in D_{E}$. Further, assume that: 
(a) each function $f_{i}, i \in I$, is E-convex at $\bar{y}$ on $D_{E} \cup Y_{\mathrm{MSD}_{E}}$,

(b) each function $-q_{i}, i \in I$, is E-convex at $\bar{y}$ on $D_{E} \cup Y_{\mathrm{MSD}_{E}}$,

(c) each function $g_{j}, j \in J_{E}(\bar{y})$, is E-convex at $\bar{y}$ on $D_{E} \cup Y_{\mathrm{MSD}_{E}}$,

(d) each function $h_{k}, k \in K_{E}^{+}(\bar{y})$, is E-convex at $\bar{y}$ on $D_{E} \cup Y_{\mathrm{MSD}_{E}}$,

(e) each function $-h_{k}, k \in K_{E}^{-}(\bar{y})$, is E-convex at $\bar{y}$ on $D_{E} \cup Y_{\mathrm{MSD}_{E}}$.

Then $\bar{y}$ is a weak Pareto solution (a Pareto solution) of the problem $\left(\mathrm{MFP}_{E}\right)$.

Proof The proof of this theorem follows directly from weak duality (Theorem 20 (Theorem 22)).

Theorem 27 (Schaible converse $E$-duality for (MFP) and $\left.\left(\mathrm{MSD}_{E}\right)\right) \operatorname{Let}\left(E(\bar{y}), \bar{v}^{E}, \bar{\lambda}, \bar{\mu}, \bar{\xi}\right) \in$ $\Omega_{\mathrm{MSD}_{E}}$ be a weakly E-efficient solution (an E-efficient solution) of a maximum type in the problem (MSD) and $E(\bar{y}) \in D$. Further, assume that all hypotheses of Theorem 26 are fulfilled. Then $E(\bar{y})$ is a weak E-Pareto solution (an E-Pareto solution) of the problem (MFP).

\section{Bector E-duality}

In this section, we extend the dual problem defined by Bector [3] for a nonlinear differentiable scalar fractional problem to the case of an $E$-differentiable multiobjective fractional programming problem with both inequality and equality constraints. Namely, we define the vector dual problem $\left(\mathrm{BFD}_{E}\right)$ in the sense of Bector [3] for the multiobjective fractional $E$-programming problem $\left(\mathrm{MFP}_{E}\right)$ and we prove several Bector duality results between $\left(\mathrm{MFP}_{E}\right)$ and $\left(\mathrm{BFD}_{E}\right)$ under appropriate $E$-convexity hypotheses. Thus, we formulate the vector $E$-dual problem $\left(\mathrm{BFD}_{E}\right)$ in the sense of Bector [3] for the considered multiobjective fractional programming problem (MFP). Further, we use Bector duality results established between $\left(\mathrm{MFP}_{E}\right)$ and $\left(\mathrm{BFD}_{E}\right)$ in proving the so-called $E$-duality results in the sense of Bector between (MFP) and $\left(\mathrm{BFD}_{E}\right)$.

Let $E: R^{n} \rightarrow R^{n}$ be a given one-to-one and onto operator. Now, we define the vector fractional dual problem $\left(\mathrm{BFD}_{E}\right)$ in the sense of Bector [3] for $\left(\mathrm{MFP}_{E}\right)$ (at the same time, the vector fractional $E$-dual problem in the sense of Bector [3] for (MFP)) as follows:

$$
\max \left(\frac{f_{1}(E(y))+\mu g(E(y))+\xi h(E(y))}{q_{1}(E(y))}, \ldots, \frac{f_{p}(E(y))+\mu g(E(y))+\xi h(E(y))}{q_{p}(E(y))}\right)
$$

subject to

$$
\begin{aligned}
& \sum_{i=1}^{p} \lambda_{i} q_{i}(E(y))\left[\nabla f_{i}(E(y))+\sum_{j=1}^{m} \mu_{j} \nabla g_{j}(E(y))+\sum_{k=1}^{r} \xi_{k} \nabla h_{k}(E(y))\right] \\
& \quad+\sum_{i=1}^{p} \lambda_{i}\left(-\nabla q_{i}(E(y))\right)\left[f_{i}(E(y))+\sum_{j=1}^{m} \mu_{j} g_{j}(E(y))+\sum_{k=1}^{r} \xi_{k} h_{k}(E(y))\right]=0 \\
& \sum_{j=1}^{m} \mu_{j} g_{j}(E(y))+\sum_{k=1}^{r} \xi_{k} h_{k}(E(y)) \geqq 0, \quad\left(\mathrm{BFD}_{E}\right) \\
& y \in R^{n}, \quad v \in R_{+}^{p}, \quad \lambda \geq 0, \quad \sum_{i=1}^{k} \lambda_{i}=1, \quad \mu \in R_{+}^{m}, \quad \xi \in R^{r} .
\end{aligned}
$$

Let $\Omega_{\mathrm{BFD}_{E}}$ denote the set of all feasible points of $\left(\mathrm{BFD}_{E}\right)$ and $\mathrm{pr}_{R^{n}} W_{\mathrm{BFD}_{E}}$ the projection of the set $\Omega_{\mathrm{BFD}_{E}}$ on $R^{n}$, that is, $\operatorname{pr}_{R^{n}} W_{\mathrm{BFD}_{E}}=\left\{y \in R^{n}: \exists \lambda \in R_{+}^{p}, \mu \in R_{+}^{m}\right.$, 
$\xi \in R^{r}$ such that $\left.(y, \lambda, \mu, \xi) \in W_{\mathrm{BFD}_{E}}\right\}$. Let us denote $Y_{\mathrm{BFD}_{E}}=\operatorname{pr}_{R^{n}} W_{\mathrm{BFD}_{E}}$ and, moreover, $\Psi(z, \mu, \xi)=\left(\Psi_{1}(z, \mu, \xi), \ldots, \Psi_{p}(z, \mu, \xi)\right), \quad z \in R^{n}$, where $\Psi_{i}^{E}(z, \mu, \xi)=f_{i}(E(z))+$ $\sum_{j=1}^{m} \mu_{j} g_{j}(E(z))+\sum_{k=1}^{r} \xi_{k} h_{k}(E(z)), i \in I$. Hence, the objective function and the first constraint of $\left(\mathrm{BFD}_{E}\right)$ can be re-written as follows:

$$
\begin{aligned}
& \max \quad\left(\frac{\Psi_{1}^{E}(y, \mu, \xi)}{q_{1}(E(y))}, \ldots, \frac{\Psi_{p}^{E}(y, \mu, \xi)}{q_{p}(E(y))}\right) \\
& \text { subject to } \sum_{i=1}^{p} \lambda_{i} q_{i}(E(y)) \nabla \Psi_{i}^{E}(y, \mu, \xi)+\sum_{i=1}^{p} \lambda_{i}\left(-\nabla q_{i}(E(y))\right) \Psi_{i}^{E}(y, \mu, \xi)=0 .
\end{aligned}
$$

Theorem 28 (Bector weak duality between $\left(\mathrm{MFP}_{E}\right)$ and $\left.\left(\mathrm{BFD}_{E}\right)\right)$ Let $x$ and $(y, \lambda, \mu, \xi)$ be any feasible solutions of the problems $\left(\mathrm{MFP}_{E}\right)$ and $\left(\mathrm{BFD}_{E}\right)$, respectively. Further, assume that one of the following hypotheses is fulfilled:

(A) (a) each function $f_{i}, i \in I$, is E-convex at $y$ on $D_{E} \cup Y_{\mathrm{BFD}_{E}}$,

(b) each function $-q_{i}, i \in I$, is E-convex at y on $D_{E} \cup Y_{\mathrm{BFD}_{E}}$,

(c) each function $g_{j}, j \in J_{E}(y)$, is E-convex at $y$ on $D_{E} \cup Y_{\mathrm{BFD}_{E}}$,

(d) each function $h_{k}, k \in K_{E}^{+}(y)$, is E-convex at $y$ on $D_{E} \cup Y_{\mathrm{BFD}_{E}}$,

(e) each function $-h_{k}, k \in K_{E}^{-}(y)$, is E-convex at $y$ on $D_{E} \cup Y_{\mathrm{BFD}_{E}}$;

(B) each function $\sum_{i=1}^{p} \lambda_{i} q_{i}(E(y)) \Psi_{i}^{E}(\cdot, \mu, \xi)+\sum_{i=1}^{p} \lambda_{i}\left(-q_{i}(E(\cdot))\right) \Psi_{i}^{E}(y, \mu, \xi)$ is E-convex at $y$ on $D_{E} \cup Y_{\mathrm{BFD}_{E}}$.

Then $\left(\frac{f_{1}(E(x))}{q_{1}(E(x))}, \ldots, \frac{f_{p}(E(x))}{q_{p}(E(x))}\right) \nless\left(\frac{\Psi_{1}^{E}(y, \mu, \xi)}{q_{1}(E(y))}, \ldots, \frac{\Psi_{p}^{E}(y, \mu, \xi)}{q_{p}(E(y))}\right)$.

Proof Let $x$ and $(y, \lambda, \mu, \xi)$ be any feasible solutions of the problems $\left(\mathrm{MFP}_{E}\right)$ and $\left(\mathrm{BFD}_{E}\right)$, respectively.

We proceed by contradiction. Suppose, contrary to the result, that

$$
\left(\frac{f_{1}(E(x))}{q_{1}(E(x))}, \ldots, \frac{f_{p}(E(x))}{q_{p}(E(x))}\right)<\left(\frac{\Psi_{1}^{E}(y, \mu, \xi)}{q_{1}(E(y))}, \ldots, \frac{\Psi_{p}^{E}(y, \mu, \xi)}{q_{p}(E(y))}\right)
$$

Hence, the inequalities above yield

$$
\frac{f_{i}(E(x))}{q_{i}(E(x))}-\frac{f_{i}(E(y))}{q_{i}(E(y))}<\frac{\sum_{j=1}^{m} \mu_{j} g_{j}(E(y))+\sum_{k=1}^{r} \xi_{k} h_{k}(E(y))}{q_{i}(E(y))}, \quad i=1, \ldots, p .
$$

Thus,

$$
\begin{aligned}
& f_{i}(E(x)) q_{i}(E(y))-f_{i}(E(y)) q_{i}(E(x)) \\
& \quad<q_{i}(E(x))\left[\sum_{j=1}^{m} \mu_{j} g_{j}(E(y))+\sum_{k=1}^{r} \xi_{k} h_{k}(E(y))\right], \quad i=1, \ldots, p .
\end{aligned}
$$

The proof of this theorem under hypothesis (A).

Using hypotheses (A)(a)-(d), by Proposition 5, we see that the inequalities

$$
\begin{aligned}
& f_{i}(E(x))-f_{i}(E(y)) \geqq \nabla f_{i}(E(y))(E(x)-E(y)), \quad i \in I, \\
& -q_{i}(E(x))+q_{i}(E(y)) \geqq-\nabla q_{i}(E(y))(E(x)-E(y)), \quad i \in I,
\end{aligned}
$$




$$
\begin{aligned}
& g_{j}(E(x))-g_{j}(E(y)) \geqq \nabla g_{j}(E(y))(E(x)-E(y)), \quad j \in J_{E}(y), \\
& h_{k}(E(x))-h_{k}(E(y)) \geqq \nabla h_{k}(E(y))(E(x)-E(y)), \quad k \in K_{E}^{+}(y), \\
& -h_{k}(E(x))+h_{k}(E(y)) \geqq-\nabla h_{k}(E(y))(E(x)-E(y)), \quad k \in K_{E}^{-}(y)
\end{aligned}
$$

hold. Multiplying each inequality (44) by $q_{i}(E(y))>0, i \in I$, and each inequality (45) by $f_{i}(E(y)) \geqq 0, i \in I$, we get, respectively,

$$
\begin{aligned}
& f_{i}(E(x)) q_{i}(E(y))-f_{i}(E(y)) q_{i}(E(y)) \geqq q_{i}(E(y)) \nabla f_{i}(E(y))(E(x)-E(y)), \quad i \in I, \\
& -f_{i}(E(y)) q_{i}(E(x))+f_{i}(E(y)) q_{i}(E(y)) \geqq f_{i}(E(y))\left(-\nabla q_{i}(E(y))\right)(E(x)-E(y)), \quad i \in I .
\end{aligned}
$$

Combining (49) and (50), we get

$$
\begin{aligned}
& f_{i}(E(x)) q_{i}(E(y))-f_{i}(E(y)) q_{i}(E(x)) \\
& \quad \geqq\left[q_{i}(E(y)) \nabla f_{i}(E(y))-f_{i}(E(y)) \nabla q_{i}(E(y))\right](E(x)-E(y)), \quad i \in I .
\end{aligned}
$$

By the second constraint of $\left(\mathrm{BFD}_{E}\right),(45)$ yields

$$
\begin{aligned}
& {\left[\sum_{j=1}^{m} \mu_{j} g_{j}(E(y))+\sum_{k=1}^{r} \xi_{k} h_{k}(E(y))\right]\left[-q_{i}(E(x))+q_{i}(E(y))\right]} \\
& \quad \geqq-\left[\sum_{j=1}^{m} \mu_{j} g_{j}(E(y))+\sum_{k=1}^{r} \xi_{k} h_{k}(E(y))\right] \nabla q_{i}(E(y))(E(x)-E(y)), \quad i \in I .
\end{aligned}
$$

Multiplying each inequality (46)-(48) by the corresponding Lagrange multiplier and then adding both sides of the resulting inequalities, we get

$$
\begin{gathered}
\sum_{j=1}^{m} \mu_{j} g_{j}(E(x))+\sum_{k=1}^{r} \xi_{k} h_{k}(E(x))-\left[\sum_{j=1}^{m} \mu_{j} g_{j}(E(y))+\sum_{k=1}^{r} \xi_{k} h_{k}(E(y))\right] \\
\geqq\left[\sum_{j=1}^{m} \mu_{j} \nabla g_{j}(E(y))+\sum_{k=1}^{r} \xi_{k} \nabla h_{k}(E(y))\right](E(x)-E(y)) .
\end{gathered}
$$

Multiplying the inequality above by $q_{i}(E(y)) \geqq 0, i \in I$, we obtain, for any $i \in I$,

$$
\begin{aligned}
q_{i}(E(y)) & {\left[\sum_{j=1}^{m} \mu_{j} g_{j}(E(x))+\sum_{k=1}^{r} \xi_{k} h_{k}(E(x))\right] } \\
& -q_{i}(E(y))\left[\sum_{j=1}^{m} \mu_{j} g_{j}(E(y))+\sum_{k=1}^{r} \xi_{k} h_{k}(E(y))\right] \\
\geqq & q_{i}(E(y))\left[\sum_{j=1}^{m} \mu_{j} \nabla g_{j}(E(y))+\sum_{k=1}^{r} \xi_{k} \nabla h_{k}(E(y))\right](E(x)-E(y)) .
\end{aligned}
$$


Combining (52) and (53), we have

$$
\begin{aligned}
& \left(-q_{i}(E(x))\right)\left[\sum_{j=1}^{m} \mu_{j} g_{j}(E(y))+\sum_{k=1}^{r} \xi_{k} h_{k}(E(y))\right] \\
& \quad+q_{i}(E(y))\left[\sum_{j=1}^{m} \mu_{j} g_{j}(E(x))+\sum_{k=1}^{r} \xi_{k} h_{k}(E(x))\right] \\
& \geqq\left(-\nabla q_{i}(E(y))\right)\left[\sum_{j=1}^{m} \mu_{j} g_{j}(E(y))+\sum_{k=1}^{r} \xi_{k} h_{k}(E(y))\right](E(x)-E(y)) \\
& \quad+q_{i}(E(y))\left[\sum_{j=1}^{m} \mu_{j} \nabla g_{j}(E(y))+\sum_{k=1}^{r} \xi_{k} \nabla h_{k}(E(y))\right](E(x)-E(y)), \quad i \in I .
\end{aligned}
$$

From $x \in D_{E}$ and $(y, \lambda, \mu, \xi) \in \Omega_{\mathrm{BFD}_{E}}$, it follows that

$$
q_{i}(E(y))\left[\sum_{j=1}^{m} \mu_{j} g_{j}(E(x))+\sum_{k=1}^{r} \xi_{k} h_{k}(E(x))\right] \leqq 0 .
$$

Then, by the above inequality, (54) implies

$$
\begin{aligned}
& \left(-q_{i}(E(x))\right)\left[\sum_{j=1}^{m} \mu_{j} g_{j}(E(y))+\sum_{k=1}^{r} \xi_{k} h_{k}(E(y))\right] \\
& \geqq\left(-\nabla q_{i}(E(y))\right)\left[\sum_{j=1}^{m} \mu_{j} g_{j}(E(y))+\sum_{k=1}^{r} \xi_{k} h_{k}(E(y))\right](E(x)-E(y)) \\
& \quad+q_{i}(E(y))\left[\sum_{j=1}^{m} \mu_{j} \nabla g_{j}(E(y))+\sum_{k=1}^{r} \xi_{k} \nabla h_{k}(E(y))\right](E(x)-E(y)), \quad i \in I .
\end{aligned}
$$

Hence, (51) and (55) yield

$$
\begin{aligned}
& f_{i}(E(x)) q_{i}(E(y))-f_{i}(E(y)) q_{i}(E(x))-q_{i}(E(x))\left[\sum_{j=1}^{m} \mu_{j} g_{j}(E(y))+\sum_{k=1}^{r} \xi_{k} h_{k}(E(y))\right] \\
& \geqq\left\{q_{i}(E(y)) \nabla f_{i}(E(y))-f_{i}(E(y)) \nabla q_{i}(E(y))\right. \\
& \quad+\left(-\nabla q_{i}(E(y))\right)\left[\sum_{j=1}^{m} \mu_{j} g_{j}(E(y))+\sum_{k=1}^{r} \xi_{k} h_{k}(E(y))\right] \\
& \left.\quad+q_{i}(E(y))\left[\sum_{j=1}^{m} \mu_{j} \nabla g_{j}(E(y))+\sum_{k=1}^{r} \xi_{k} \nabla h_{k}(E(y))\right]\right\}(E(x)-E(y)), \quad i \in I .
\end{aligned}
$$

Combining (43) and (56), we have

$$
\left\{q_{i}(E(y)) \nabla f_{i}(E(y))-f_{i}(E(y)) \nabla q_{i}(E(y))\right.
$$




$$
\begin{aligned}
& +\left(-\nabla q_{i}(E(y))\right)\left[\sum_{j=1}^{m} \mu_{j} g_{j}(E(y))+\sum_{k=1}^{r} \xi_{k} h_{k}(E(y))\right] \\
& \left.+q_{i}(E(y))\left[\sum_{j=1}^{m} \mu_{j} \nabla g_{j}(E(y))+\sum_{k=1}^{r} \xi_{k} \nabla h_{k}(E(y))\right]\right\}(E(x)-E(y))<0, \quad i \in I .
\end{aligned}
$$

Hence, each inequality above can be re-written in the following form:

$$
\begin{aligned}
& \left\{\left(-\nabla q_{i}(E(y))\right)\left[f_{i}(E(y))+\sum_{j=1}^{m} \mu_{j} g_{j}(E(y))+\sum_{k=1}^{r} \xi_{k} h_{k}(E(y))\right]\right. \\
& \left.+q_{i}(E(y))\left[\nabla f_{i}(E(y))+\sum_{j=1}^{m} \mu_{j} \nabla g_{j}(E(y))+\sum_{k=1}^{r} \xi_{k} \nabla h_{k}(E(y))\right]\right\}(E(x)-E(y))<0
\end{aligned}
$$

$i \in I$.

Thus,

$$
\left[q_{i}(E(y)) \nabla \Psi_{i}(y, \mu, \xi)+\left(-\nabla q_{i}(E(y))\right) \Psi_{i}(y, \mu, \xi)\right](E(x)-E(y))<0, \quad i \in I .
$$

Multiplying each inequality above by $\lambda_{i}, i \in I$, and then adding both sides of the resulting inequalities, we see that the inequality

$$
\left[\sum_{i=1}^{p} \lambda_{i} q_{i}(E(y)) \nabla \Psi_{i}(y, \mu, \xi)+\sum_{i=1}^{p} \lambda_{i}\left(-\nabla q_{i}(E(y))\right) \Psi_{i}(y, \mu, \xi)\right](E(x)-E(y))<0
$$

holds, contradicting the first constraint of $\left(\mathrm{BFD}_{E}\right)$. This completes the proof of this theorem under hypothesis (A).

The proof of this theorem under hypothesis (B). Using hypotheses (B), by Proposition 5, we see that the inequalities

$$
\begin{aligned}
\sum_{i=1}^{p} \lambda_{i} q_{i}(E(y)) \Psi_{i}^{E}(x, \mu, \xi)+\sum_{i=1}^{p} \lambda_{i}\left(-q_{i}(E(x))\right) \Psi_{i}^{E}(y, \mu, \xi) \\
\quad-\sum_{i=1}^{p} \lambda_{i} q_{i}(E(y)) \Psi_{i}^{E}(y, \mu, \xi)+\sum_{i=1}^{p} \lambda_{i}\left(-q_{i}(E(y))\right) \Psi_{i}^{E}(y, \mu, \xi) \\
\geqq \sum_{i=1}^{p} \lambda_{i} q_{i}(E(y)) \nabla \Psi_{i}^{E}(y, \mu, \xi)+\sum_{i=1}^{p} \lambda_{i}\left(-\nabla q_{i}(E(y))\right) \Psi_{i}^{E}(y, \mu, \xi) .
\end{aligned}
$$

Thus, the first constraint of $\left(\mathrm{BFD}_{E}\right)$ implies

$$
\sum_{i=1}^{p} \lambda_{i} q_{i}(E(y)) \Psi_{i}^{E}(x, \mu, \xi) \geqq \sum_{i=1}^{p} \lambda_{i} q_{i}(E(x)) \Psi_{i}^{E}(y, \mu, \xi) .
$$


Hence, (57) gives

$$
\begin{aligned}
& \sum_{i=1}^{p} \lambda_{i} f_{i}(E(x)) q_{i}(E(y))+\sum_{i=1}^{p} \lambda_{i} q_{i}(E(y))\left[\sum_{j=1}^{m} \mu_{j} g_{j}(E(x))+\sum_{k=1}^{r} \xi_{k} h_{k}(E(x))\right] \\
& \quad \geqq \sum_{i=1}^{p} \lambda_{i} f_{i}(E(y)) q_{i}(E(x))+\sum_{i=1}^{p} \lambda_{i} q_{i}(E(x))\left[\sum_{j=1}^{m} \mu_{j} g_{j}(E(y))+\sum_{k=1}^{r} \xi_{k} h_{k}(E(y))\right] .
\end{aligned}
$$

From $x \in D_{E}$ and $(y, \lambda, \mu, \xi) \in \Omega_{\mathrm{BFD}_{E}}$, it follows that

$$
\sum_{i=1}^{p} \lambda_{i} q_{i}(E(y))\left[\sum_{j=1}^{m} \mu_{j} g_{j}(E(x))+\sum_{k=1}^{r} \xi_{k} h_{k}(E(x))\right] \leqq 0
$$

Combining (58) and (59), we get

$$
\begin{aligned}
& \sum_{i=1}^{p} \lambda_{i} f_{i}(E(x)) q_{i}(E(y))-\sum_{i=1}^{p} \lambda_{i} f_{i}(E(y)) q_{i}(E(x)) \\
& \quad \geqq \sum_{i=1}^{p} \lambda_{i} q_{i}(E(x))\left[\sum_{j=1}^{m} \mu_{j} g_{j}(E(y))+\sum_{k=1}^{r} \xi_{k} h_{k}(E(y))\right] .
\end{aligned}
$$

However, multiplying each inequality (43) by $\lambda_{i}, i \in I$, and then summing the resulting inequalities, we find that the inequality

$$
\begin{aligned}
& \sum_{i=1}^{p} \lambda_{i} f_{i}(E(x)) q_{i}(E(y))-\sum_{i=1}^{p} \lambda_{i} f_{i}(E(y)) q_{i}(E(x)) \\
& \quad<\sum_{i=1}^{p} \lambda_{i} q_{i}(E(x))\left[\sum_{j=1}^{m} \mu_{j} g_{j}(E(y))+\sum_{k=1}^{r} \xi_{k} h_{k}(E(y))\right]
\end{aligned}
$$

holds, contradicting (60). This completes the proof of this theorem under hypothe$\operatorname{sis}(\mathrm{B})$.

Theorem 29 (Bector weak $E$-duality between (MFP) and $\left.\left(\mathrm{BFD}_{E}\right)\right)$ Let $E(x)$ and $(y, \lambda, \mu, \xi)$ be any feasible solutions of the problems $(\mathrm{MFP})$ and $\left(\mathrm{BFD}_{E}\right)$, respectively. Further, assume that all hypotheses of Theorem 28 are fulfilled. Then $\left(\frac{f_{1}(E(x))}{q_{1}(E(x))}, \ldots, \frac{f_{p}(E(x))}{q_{p}(E(x))}\right) \nless\left(\frac{\Psi_{1}^{E}(y, \mu, \xi)}{q_{1}(E(y))}, \ldots\right.$, $\left.\frac{\Psi_{p}^{E}(y, \mu, \xi)}{q_{p}(E(y))}\right)$.

Proof The proof of this theorem follows directly from Theorem 28 and Proposition 11.

If we impose some stronger $E$-convexity assumption on the objective function $f$, then the following result is true.

Theorem 30 (Bector weak duality between $\left(\mathrm{MFP}_{E}\right)$ and $\left.\left(\mathrm{BFD}_{E}\right)\right)$ Let $x$ and $(y, \lambda, \mu, \xi)$ be any feasible solutions of the problems $\left(\mathrm{MFP}_{E}\right)$ and $\left(\mathrm{BFD}_{E}\right)$, respectively. Further, assume that one of the following hypotheses is fulfilled:

(A) (a) each function $f_{i}, i \in I$, is strictly E-convex at $y$ on $D_{E} \cup Y_{\mathrm{BFD}_{E}}$, 
(b) each function $-q_{i}, i \in I$, is E-convex at y on $D_{E} \cup Y_{\mathrm{BFD}_{E}}$,

(c) each function $g_{j}, j \in J_{E}(y)$, is E-convex at $y$ on $D_{E} \cup Y_{\mathrm{BFD}_{E}}$,

(d) each function $h_{k}, k \in K_{E}^{+}(y)$, is E-convex at $y$ on $D_{E} \cup Y_{\mathrm{BFD}_{E}}$,

(e) each function $-h_{k}, k \in K_{E}^{-}(y)$, is E-convex at $y$ on $D_{E} \cup Y_{\mathrm{BFD}_{E}}$;

(B) each function $\sum_{i=1}^{p} \lambda_{i} q_{i}(E(y)) \Psi_{i}^{E}(\cdot, \mu, \xi)+\sum_{i=1}^{p} \lambda_{i}\left(-q_{i}(E(\cdot))\right) \Psi_{i}^{E}(y, \mu, \xi)$ is strictly

E-convex at $y$ on $D_{E} \cup Y_{\mathrm{BFD}_{E}}$.

Then $\left(\frac{f_{1}(E(x))}{q_{1}(E(x))}, \ldots, \frac{f_{p}(E(x))}{q_{p}(E(x))}\right) \not \leq\left(\frac{\Psi_{1}^{E}(y, \mu, \xi)}{q_{1}(E(y))}, \ldots, \frac{\Psi_{p}^{E}(y, \mu, \xi)}{q_{p}(E(y))}\right)$.

Theorem 31 (Stronger Bector weak $E$-duality between (MFP) and $\left.\left(\mathrm{BFD}_{E}\right)\right)$ Let $E(x)$ and $(y, \lambda, \mu, \xi)$ be any feasible solutions of the problems (MFP) and $\left(\mathrm{BFD}_{E}\right)$, respectively. Further, assume that all hypotheses of Theorem 30 are fulfilled. Then $\left(\frac{f_{1}(E(x))}{q_{1}(E(x))}, \ldots, \frac{f_{p}(E(x))}{q_{p}(E(x))}\right) \not \leq$ $\left(\frac{\Psi_{1}^{E}(y, \mu, \xi)}{q_{1}(E(y))}, \ldots, \frac{\Psi_{p}^{E}(y, \mu, \xi)}{q_{p}(E(y))}\right)$.

Now, we prove Bector strong duality between $\left(\mathrm{MFP}_{E}\right)$ and $\left(\mathrm{BFD}_{E}\right)$ which we use in proving Bector strong $E$-duality between (MFP) and $\left(\mathrm{BFD}_{E}\right)$.

Theorem 32 (Bector strong duality between $\left(\mathrm{MFP}_{E}\right)$ and $\left.\left(\mathrm{BFD}_{E}\right)\right)$ Let $\bar{x}$ be a weak Pareto solution (a Pareto solution) of the multiobjective fractional E-programming problem $\left(\mathrm{MFP}_{E}\right)$ and the constraint qualification [1] be satisfied at $\bar{x}$. Then there exist $\bar{\lambda} \in R_{+}^{p}$, $\bar{\mu} \in R_{+}^{m}$ and $\bar{\xi} \in R^{r}$ such that $(\bar{x}, \bar{\lambda}, \bar{\mu}, \bar{\xi})$ is feasible in the Bector dual problem $\left(\mathrm{BFD}_{E}\right)$ and

$$
\frac{f(E(\bar{x}))}{q(E(\bar{x}))}=\left(\frac{\Psi_{1}^{E}(\bar{x}, \bar{\mu}, \bar{\xi})}{q_{1}(E(\bar{x}))}, \ldots, \frac{\Psi_{p}^{E}(\bar{x}, \bar{\mu}, \bar{\xi})}{q_{p}(E(\bar{x}))}\right)
$$

If also all hypotheses of the weak duality theorem-Theorem 28 (Theorem 30)-are satisfied, then $(\bar{x}, \bar{\lambda}, \bar{\mu}, \bar{\xi})$ is a weakly efficient solution (an efficient solution) of a maximum type for the problem $\left(\mathrm{BFD}_{E}\right)$.

Proof By assumption, $\bar{x}$ is a weak Pareto solution (a Pareto solution) of the problem $\left(\mathrm{MFP}_{E}\right)$ and the suitable constraint qualification [1] is satisfied at $\bar{x}$. Then, by $\bar{x} \in D_{E}$ and Theorem 14, the necessary optimality conditions (7)-(9) are fulfilled. If we put $\bar{v}^{E}=\frac{f(E(\bar{x}))}{q(E(\bar{x}))}$ in (7), then we get

$$
\begin{aligned}
& \sum_{i=1}^{p} \bar{\lambda}_{i}\left(\nabla f_{i}(E(\bar{x})) q_{i}(E(\bar{x}))-f_{i}(E(\bar{x})) \nabla q_{i}(E(\bar{x}))\right) \\
& \quad+q_{i}(E(\bar{x}))\left[\sum_{j=1}^{m} \bar{\mu}_{j} \nabla g_{j}(E(\bar{x}))+\sum_{k=1}^{r} \bar{\xi}_{k} \nabla h_{k}(E(\bar{x}))\right]=0 .
\end{aligned}
$$

By the necessary optimality condition (8) and $\bar{x} \in D_{E}$, it follows that

$$
\sum_{j=1}^{m} \bar{\mu}_{j} g_{j}(E(\bar{x}))+\sum_{k=1}^{r} \bar{\xi}_{k} h_{k}(E(\bar{x}))=0 .
$$


By the necessary optimality condition (9), (62) implies

$$
\begin{aligned}
& \sum_{i=1}^{p} \bar{\lambda}_{i}\left(-\nabla q_{i} E(\bar{x})\right)\left[f_{i}(E(\bar{x}))+\sum_{j=1}^{m} \bar{\mu}_{j} g_{j}(E(\bar{x}))+\sum_{k=1}^{r} \bar{\xi}_{k} h_{k}(E(\bar{x}))\right] \\
& \quad+\sum_{i=1}^{p} \bar{\lambda}_{i} q_{i}(E(\bar{x}))\left[\nabla f_{i}(E(\bar{x}))+\sum_{j=1}^{m} \bar{\mu}_{j} \nabla g_{j}(E(\bar{x}))+\sum_{k=1}^{r} \bar{\xi}_{k} \nabla h_{k}(E(\bar{x}))\right]=0 .
\end{aligned}
$$

Hence, by (63), (62) and the necessary optimality condition (9), we conclude that $(\bar{x}, \bar{\lambda}, \bar{\mu}, \bar{\xi})$ is feasible in $\left(\mathrm{BFD}_{E}\right)$. Obviously, the corresponding objective function value in $\left(\mathrm{BFD}_{E}\right)$ is equal to $\frac{f(E(\bar{x}))}{q(E(\bar{x}))}$. Let $(y, \lambda, \mu, \xi)$ be any feasible solution of $\left(\mathrm{BFD}_{E}\right)$. Then, by the weak duality theorem-Theorem 28 (Theorem 30), it follows that the inequality

$$
\left(\frac{\Psi_{1}^{E}(\bar{x}, \bar{\mu}, \bar{\xi})}{q_{1}(E(\bar{x}))}, \ldots, \frac{\Psi_{p}^{E}(\bar{x}, \bar{\mu}, \bar{\xi})}{q_{p}(E(\bar{x}))}\right) \nless\left(\frac{\Psi_{1}^{E}(y, \mu, \xi)}{q_{1}(E(y))}, \ldots, \frac{\Psi_{p}^{E}(y, \mu, \xi)}{q_{p}(E(y))}\right)
$$

holds. This implies that $(\bar{x}, \bar{\lambda}, \bar{\mu}, \bar{\xi})$ is a weakly efficient solution (an efficient solution) of a maximum type of the problem $\left(\mathrm{BFD}_{E}\right)$. This completes the proof of this theorem.

Theorem 33 (Bector strong E-duality between (MFP) and $\left.\left(\mathrm{BFD}_{E}\right)\right)$ Let $E(\bar{x})$ be a weak E-Pareto solution (an E-Pareto solution) of the multiobjective fractional E-programming problem (MFP) and the constraint qualification [1] be satisfied. Then, there exist $\bar{\lambda} \in R_{+}^{p}$, $\bar{\mu} \in R_{+}^{m}$ and $\bar{\xi} \in R^{r}$ such that $(\bar{x}, \bar{\lambda}, \bar{\mu}, \bar{\xi})$ is feasible in the Bector dual problem $\left(\mathrm{BFD}_{E}\right)$ and

$$
\frac{f(E(\bar{x}))}{q(E(\bar{x}))}=\left(\frac{\Psi_{1}^{E}(\bar{x}, \bar{\mu}, \bar{\xi})}{q_{1}(E(\bar{x}))}, \ldots, \frac{\Psi_{p}^{E}(\bar{x}, \bar{\mu}, \bar{\xi})}{q_{p}(E(\bar{x}))}\right)
$$

If also all hypotheses of the weak duality theorem-Theorem 29 (Theorem 31)-are satisfied, then $(\bar{x}, \bar{\lambda}, \bar{\mu}, \bar{\xi})$ is a weakly E-efficient solution (an E-efficient solution) of a maximum type for the problem $\left(\mathrm{BFD}_{E}\right)$.

Remark 34 In fact, (63), (62) and (9) are the parameter-free necessary optimality conditions for $\bar{x} \in D_{E}$ to be a weak Pareto solution (a Pareto solution) of the multiobjective fractional $E$-programming problem $\left(\mathrm{MFP}_{E}\right)$. At the same time, (63), (62) and (9) are the parameter-free necessary $E$-optimality conditions for $E(\bar{x}) \in D$ to be a weak $E$-Pareto solution (an $E$-Pareto solution) of the considered multiobjective fractional programming problem (MFP).

Theorem 35 (Bector converse duality between $\left(\mathrm{MFP}_{E}\right)$ and $\left.\left(\mathrm{BFD}_{E}\right)\right) \operatorname{Let}(\bar{y}, \bar{\lambda}, \bar{\mu}, \bar{\xi}) \in$ $\Omega_{\mathrm{BFD}_{E}}$ be a weakly efficient solution (an efficient solution) of a maximum type in the problem $\left(\mathrm{BFD}_{E}\right)$ and $\bar{y} \in D_{E}$. Further, assume that:

(a) each function $f_{i}, i \in I$, is E-convex at $\bar{y}$ on $D_{E} \cup Y_{\mathrm{BFD}_{E}}$,

(b) each function $-q_{i}, i \in I$, is E-convex at $\bar{y}$ on $D_{E} \cup Y_{\mathrm{BFD}_{E}}$,

(c) each function $g_{j}, j \in J_{E}(\bar{y})$, is E-convex at $\bar{y}$ on $D_{E} \cup Y_{\mathrm{BFD}_{E}}$,

(d) each function $h_{k}, k \in K_{E}^{+}(\bar{y})$, is E-convex at $\bar{y}$ on $D_{E} \cup Y_{\mathrm{BFD}_{E}}$,

(e) each function $-h_{k}, k \in K_{E}^{-}(\bar{y})$, is E-convex at $\bar{y}$ on $D_{E} \cup Y_{\mathrm{BFD}_{E}}$.

Then $\bar{y}$ is a weak Pareto solution (a Pareto solution) of the problem $\left(\mathrm{MFP}_{E}\right)$. 
Proof The proof of theorem follows directly from weak duality-Theorem 28 (Theorem 30).

Theorem 36 (Bector converse $E$-duality for (MFP) and $\left.\left(\mathrm{BFD}_{E}\right)\right)$ Let $(E(\bar{y}), \bar{\lambda}, \bar{\mu}, \bar{\xi}) \in \Omega_{\mathrm{BFD}_{E}}$ be a weakly E-efficient solution (an E-efficient solution) of a maximum type in the problem $\left(\mathrm{BFD}_{E}\right)$ and $E(\bar{y}) \in D$. Further, assume that all hypotheses of Theorem 35 are fulfilled. Then $E(\bar{x})$ is a weak E-Pareto solution (an E-Pareto solution) of the problem (MFP).

\section{Conclusions}

In the paper, the class of $E$-differentiable multiobjective fractional programming problems with both inequality and equality constraints has been considered. The so-called $E$-Karush-Kuhn-Tucker necessary optimality conditions and, under appropriate $E$ convexity hypotheses, sufficient $E$-optimality conditions for weakly $E$-efficiency ( $E$-efficiency) have been established in the paper for such a class of (not necessarily) differentiable multiobjective fractional programming problems. Further, for the considered $E$-differentiable multiobjective fractional programming problem, its parametric vector $E$-dual problem in the sense of Schaible and its nonparametric multiobjective fractional $E$-dual problem in the sense of Bector have been defined. Then, also under appropriate $E$-convexity hypotheses, various $E$-duality results have been proved between this (not necessarily) differentiable multiobjective fractional programming problem and aforesaid vector $E$-duals.

To the best of our knowledge, all optimality and duality results established in this paper for the considered class of nonconvex vector fractional optimization problems with $E$ differentiable $E$-convex functions are new in the area of multiobjective fractional programming. In our opinion, the approach suggested in this paper can be extended for proving the similar $E$-optimality and $E$-duality results for other classes of fractional programming problems with $E$-differentiable functions. This may be the topic of some of our forthcoming papers.

\section{Funding}

Not applicable.

Competing interests

The authors declare that they have no competing interests.

Authors' contributions

Both authors contributed equally and significantly in writing this article. Both authors read and approved the final manuscript.

\section{Author details}

${ }^{1}$ Faculty of Mathematics and Computer Science, University of Łódź, Łódź, Poland. ²Department of Mathematics, Hadhramout University, Al-Mahrah, Yemen.

\section{Publisher's Note}

Springer Nature remains neutral with regard to jurisdictional claims in published maps and institutional affiliations.

Received: 12 August 2019 Accepted: 28 October 2019 Published online: 13 November 2019

\section{References}

1. Antczak, T., Abdulaleem, N.: E-Optimality conditions and Wolfe $E$-duality for $E$-differentiable vector optimization problems with inequality and equality constraints. J. Nonlinear Sci. Appl. 12, 745-764 (2019)

2. Antczak, T., Verma, R.: Parametric nondifferentiable multiobjective fractional programming under $(b, \Psi, \Phi, \rho)$-univexity. Turk. J. Math. 42, 2125-2147 (2018)

3. Bector, C.R.: Duality in nonlinear fractional programming. Z. Oper--Res. 17, 183-193 (1973) 
4. Bector, C.R., Chandra, S., Husain, l.: Optimality condition and duality in subdifferentiable multiobjective fractional programming. J. Optim. Theory Appl. 79, 105-125 (1993)

5. Chen, X:: Optimality and duality for the multiobjective fractional programming with the generalized $(F, \rho)$-convexity. J. Math. Anal. Appl. 273, 190-205 (2002)

6. Chinchuluun, A., Yuan, D.H., Pardalos, P.M.: Optimality conditions and duality for nondifferentiable multiobjective fractional programming with generalized convexity. Ann. Oper. Res. 154, 133-147 (2007)

7. Crouzeix, J.P., Ferland, J.A., Schaible, S.: Duality in generalized fractional programming. Math. Program. 27, 343-354 (1983)

8. Crouzeix, J.P., Ferland, J.A., Schaible, S.: An algorithm for generalized fractional programs. J. Optim. Theory Appl. 47, 35-49 (1985)

9. Dinkelbach, W.: On nonlinear fractional programming. Management Sci. 13, 492-498 (1967)

10. Dubey, R., Gupta, K.S.: On duality for a second-order multiobjective fractional programming problem involving type-1 functions. Georgian Math. J. 26, 393-404 (2019). https://doi.org/10.1515/gmj-2017-0038

11. Egudo, R.R.: Multiobjective fractional duality. Bull. Aust. Math. Soc. 37, 367-378 (1988)

12. Ho, S.-Ch.: Saddle point criteria in multiobjective fractional programming involving exponential invexity. Bull. Malays. Math. Soc. 41, 1923-1934 (2018)

13. Jagannathan, R.: On some properties of programming problems in parametric form pertaining to fractional programming. Management Sci. 12, 609-615 (1966)

14. Jayswal, A., Ahmad, I., Prasad, A.K.: Duality in multiobjective fractional programming problems involving $\left(H_{p}, r\right)$-invex functions. J. Appl. Math. Inform. 32, 99-111 (2014)

15. Jayswal, A., Kumar, R., Kumar, D.: Multiobjective fractional programming problems involving $(p, r)-\rho-(\eta, \theta)$-invex function. J. Appl. Math. Comput. 39, 35-51 (2012)

16. Jayswal, A., Stancu-Minasian, I., Stancu, A.M.: Multiobjective fractional programming problems involving semilocally type-I univex functions. Southeast Asian Bull. Math. 38, 225-241 (2014)

17. Kim, D.S.: Nonsmooth multiobjective fractional programming with generalized invexity. Taiwan. J. Math. 10, 467-478 (2006)

18. Kim, D.S., Kim, S.J., Kim, M.H.: Optimality and duality for a class of nondifferentiable multiobjective fractional programming problems. J. Optim. Theory Appl. 129, 131-146 (2006)

19. Kuk, H., Kim, H.-J.: Optimality conditions and duality for nonsmooth generalized fractional programming problems. J. Stat. Manag. Syst. 6, 207-215 (2003)

20. Kuk, H., Lee, G.M., Tanino, T:: Optimality and duality for nonsmooth multiobjective fractional programming with generalized invexity. J. Math. Anal. Appl. 262, 365-375 (2001)

21. Lee, J., Ho, S.: Optimality and duality for multiobjective fractional problems with $r$-invexity. Taiwan. J. Math. 12 719-740 (2008)

22. Liang, Z.A., Huang, H.X., Pardalos, P.M.: Optimality conditions and duality for a class of nonlinear fractional programming problems. J. Optim. Theory Appl. 110, 611-619 (2001)

23. Liang, Z.A., Huang, H.X., Pardalos, P.M.: Efficiency conditions and duality for a class of multiobjective fractional programming problems. J. Glob. Optim. 27, 447-471 (2003)

24. Liu, J.C.: Optimality and duality for multiobjective fractional programming involving nonsmooth $(F, \rho)$-convex functions. Optimization 36, 333-346 (1996)

25. Liu, J.C.: Optimality and duality for multiobjective fractional programming involving nonsmooth pseudoinvex functions. Optimization 37, 27-39 (1996)

26. Liu, S., Feng, E.: Optimality conditions and duality for a class of nondifferentiable multi-objective fractional programming problems. J. Glob. Optim. 38, 653-666 (2007)

27. Long, X.J.: Optimality conditions and duality for nondifferentiable multiobjective fractional programming problems with $(C, \alpha, \rho, d)$-convexity. J. Optim. Theory Appl. 148, 197-208 (2011)

28. Long, X.J., Huang, N.J., Liu, Z.B.: Optimality conditions, duality and saddle points for nondifferentiable multiobjective fractional programs. J. Ind. Manag. Optim. 4, 287-298 (2008)

29. Megahed, A.A., Gomma, H.G., Youness, E.A., El-Banna, A.H.: Optimality conditions of E-convex programming for an E-differentiable function. J. Inequal. Appl. 2013, Article ID 246 (2013)

30. Mukherjee, R.N.: Generalized convex duality for multiobjective fractional programs. J. Math. Anal. Appl. 162, 309-316 (1991)

31. Mukherjee, R.N., Purnachandra Rao, Ch.: Multiobjective fractional programming under generalized invexity. Indian 」. Pure Appl. Math. 27, 1175-1183 (1996)

32. Nobakhtian, S.: Optimality and duality for nonsmooth multiobjective fractional programming with mixed constraints. J. Glob. Optim. 41, 103-115 (2008)

33. Osuna-Gómez, R., Rufián-Lizana, A., Ruiz-Canales, P.: Multiobjective fractional programming with generalized convexity. Top 8, 97-110 (2000)

34. Schaible, S.: Fractional programming. I, duality. Manag. Sci. 22, 858-867 (1976)

35. Stancu-Minasian, I.M.: Fractional Programming: Theory, Methods and Applications. Kluwer Academic, Dordrecht (1997)

36. Stancu-Minasian, I.M.: A eighth bibliography of fractional programming. Optimization 66, 439-470 (2017)

37. Suneja, S.K., Lalitha, C.S.: Multiobjective fractional programming involving $\rho$-invex and related functions. Opsearch 30,1-14 (1993)

38. Verma, R.U., Seol, Y.: Some sufficient efficiency conditions in semiinfinite multiobjective fractional programming based on exponential type invexities. J. Inequal. Appl. 2015, Article ID 252 (2015)

39. Weir, T: A duality theorem for a multiple objective fractional optimization problem. Bull. Aust. Math. Soc. 34, 415-425 (1986)

40. Youness, E.A.: E-Convex sets, E-convex functions, and E-convex programming. J. Optim. Theory Appl. 102, 439-450 (1999)

41. Zalmai, G.J.: Optimality conditions and duality models for generalized fractional programming problems containing locally subdifferentiable and $\rho$-convex functions. Optimization 32, 95-124 (1995) 
42. Zezheng, W., Fenghua, Z:: Optimality and duality for a class of nonlinear fractional programming problems. J Sichuan Norm. Univ. 30, 594-597 (2007)

43. Zhang, X., Wu, Z:: Optimality conditions and duality of three kinds of nonlinear fractional programming problems Adv. Oper. Res. 2013, Article ID 708979 (2013)

Submit your manuscript to a SpringerOpen ${ }^{\circ}$ journal and benefit from:

- Convenient online submission

- Rigorous peer review

- Open access: articles freely available online

- High visibility within the field

- Retaining the copyright to your article

Submit your next manuscript at $\boldsymbol{s p r i n g e r o p e n . c o m ~}$ 